\title{
Estudio tecnológico de las estatuillas antropomorfas y de los tupus miniatura del hallazgo de Paucartambo (Cusco, Perú)
}

Étude technologique des figurines anthropomorphes et des tupus miniatures de la découverte de Paucartambo (Cusco, Pérou)

Technological study of the anthropomorphic statuettes and miniature tupus from a discovery in Paucartambo (Cusco, Peru)

Julio César Sierra Palomino y Maria Filomena Guerra

\section{(2) OpenEdition} Journals

Edición electrónica

URL: http://journals.openedition.org/bifea/8387

DOI: 10.4000/bifea.8387

ISSN: 2076-5827

\section{Editor}

Institut Français d'Études Andines

\section{Edición impresa}

Fecha de publicación: 1 abril 2017

Paginación: 151-170

ISSN: 0303-7495

Referencia electrónica

Julio César Sierra Palomino y Maria Filomena Guerra, « Estudio tecnológico de las estatuillas antropomorfas y de los tupus miniatura del hallazgo de Paucartambo (Cusco, Perú) », Bulletin de I'Institut français d'études andines [En línea], 46 (1) | 2017, Publicado el 08 abril 2017, consultado el 05 noviembre 2020. URL : http://journals.openedition.org/bifea/8387 ; DOI : https://doi.org/10.4000/ bifea.8387

\section{(c) $(1) \odot$}

Les contenus du Bulletin de l'Institut français d'études andines sont mis à disposition selon les termes de la licence Creative Commons Attribution - Pas d'Utilisation Commerciale - Pas de Modification 4.0 International. 


\title{
Estudio tecnológico de las estatuillas antropomorfas y de los tupus miniatura del hallazgo de Paucartambo (Cusco, Perú)
}

\author{
Julio César Sierra Palomino* \\ Maria Filomena Guerra**
}

\section{Resumen}

En el trabajo se describen los estudios realizados por microscopía óptica y fluorescencia de rayos $X$ (FRX) portátil a seis estatuillas y siete tupus miniatura, objetos inkas de sacrificios rituales, ofrendas en aleación de plata y en aleación de oro, provenientes del sitio arqueológico de Chimur, en Chayllabamba, provincia de Paucartambo; es parte de la colección del Museo Inka del Cusco (Perú). Este conjunto incluye la única estatuilla antropomorfa bicolor de proveniencia conocida (elaborada en plata y cobre) y una ofrenda intacta presentada en una caja de madera, conteniendo una estatuilla femenina en plata, envuelta en textiles prendidos con dos tupus de plata. Los resultados registrados sobre la diversidad tipológica de las estatuillas, la diversidad de las tecnologías y de las aleaciones empleadas por los orfebres en su elaboración son discutidos, así como la homogeneidad observada en la elaboración de los tupus.

Palabras clave: Periodo incaico, Paucartambo, estatuillas, tupus, plata, oro, aleaciones

\section{Étude technologique des figurines anthropomorphes et des tupus miniatures de la découverte de Paucartambo (Cusco, Pérou)}

\section{Résumé}

Six figurines et sept tupus miniatures destinés aux rituels de sacrifice et aux offrandes, en alliage d'argent et en alliage d'or, de la collection du musée Inka de Cusco (Pérou), attribués à la période

* Laboratorio Fisicoquímico de la Dirección Desconcentrada de Cultura de Cusco, Perú. Email: juliocsierra@yahoo.es

** CNRS, ArchAm-UMR 8096, Maison Archéologie \& Ethnologie, 21 allée de I'Université, 92023 Nanterre, France. E-mail: maria.guerra@cnrs.fr 
Inca et provenant du site archéologique de Chimur, à Chayllabamba, province de Paucartambo, sont étudiés par microscopie optique et spectrométrie par fluorescence aux rayons X (FRX) portable. Cet ensemble comprend la seule figurine anthropomorphe bicolore d'origine connue (réalisée en argent et en cuivre) ainsi qu'une offrande intacte constituée d'une boîte en bois contenant une figurine féminine en argent entourée de textiles retenus par deux tupus en argent. Les résultats obtenus sur la diversité typologique des figurines et sur les technologies et alliages employés par les orfèvres dans la fabrication des figurines sont discutés, ainsi que l'homogénéité de production des tupus.

Mots-clés : Période inca, Paucartambo, figurines, tupus, argent, or, alliages

\title{
Technological study of the anthropomorphic statuettes and miniature tupus from a discovery in Paucartambo (Cusco, Peru)
}

\begin{abstract}
Six figurines and seven miniature tupus from ritual sacrifices and offerings, made from silver alloys and gold alloys exist in the collection of the Museo Inka in Cusco (Peru). They are dated to the Inca period and were found in the archaeological site of Chimur, in Chayllabamba, district of Paucartambo. In this article, we present a study of them by optical microscopy and mobile X-ray fluorescence spectrometry (pXRF). This group of objects includes the only known two-coloured anthropomorphic figurine (made from silver and from copper) with provenience. It also includes an undamaged offering consisting of a wood box containing a silver female figurine wrapped in a cloth fastened with two silver tupus. The research results obtained on the figurines' typology and on the diversity of the technologies and alloys employed for their production are discussed, as well as the homogeneity of the tupus production.
\end{abstract}

Keywords: Inca period, Paucartambo, figurines, tupus, silver, gold, alloys

\section{INTRODUCCIÓN}

En este trabajo se estudian las estatuillas antropomorfas y algunos alfileres miniatura de pecho (tupus) del ajuar funerario de una tumba de periodo incaico encontrada en Ccorihuayrachina (Qoriwayrachina), en el sitio arqueológico de «Chimur», en la provincia de Paucartambo, departamento del Cusco, Perú (fig. 1). Este ajuar funerario fue entregado voluntariamente al Museo e Instituto Arqueológico, hoy Museo Inka, del Cusco, en septiembre-octubre del año 1947, por el señor Carlos Rodríguez que, en esta época, hizo exploraciones clandestinas de varias tumbas de la región del Cusco.

El conjunto de objetos del ajuar proviene de la excavación clandestina de una tumba de cacique, lo que restringe los datos arqueológicos asociados al hallazgo y dificulta su datación exacta. No obstante, se puede alegar que la información dada en 1947 sobre la ubicación de la tumba1 y sobre los objetos ahí encontrados es confiable.

1 Veremos más adelante la grande diversidad de las tecnologías y aleaciones empleadas en la elaboración del conjunto de estatuillas. 
Los detalles del hallazgo fueron anotados y los objetos registrados en el «Catálogo Razonado del Instituto Arqueológico del Cusco». Se proporciona el texto de la observación sobre el hallazgo anotada y firmada por el Dr. Luis A. Pardo, Director en esa época del Instituto:

Tumba de Ccorihuayrachina, lugar arqueológico Chimur, Provincia de Paucartambo. En medio de unos pajonales y donde existen grandes hendiduras de truenos por efecto del rayo, un señor Carlos Rodríguez, vecino de la región, hizo exploraciones clandestinas hallando varias tumbas, entre las cuales una que se cree ser de un Cacique, cuyo ajuar funerario, voluntariamente el citado señor Carlos Rodríguez lo trajo al museo en el año 1947 (de septiembre a octubre), en vista de que el sub-Prefecto de la provincia señor Don Pio Núñez del Prado, lo conminó y lo tomó preso en esa localidad; al salir de su reclusión

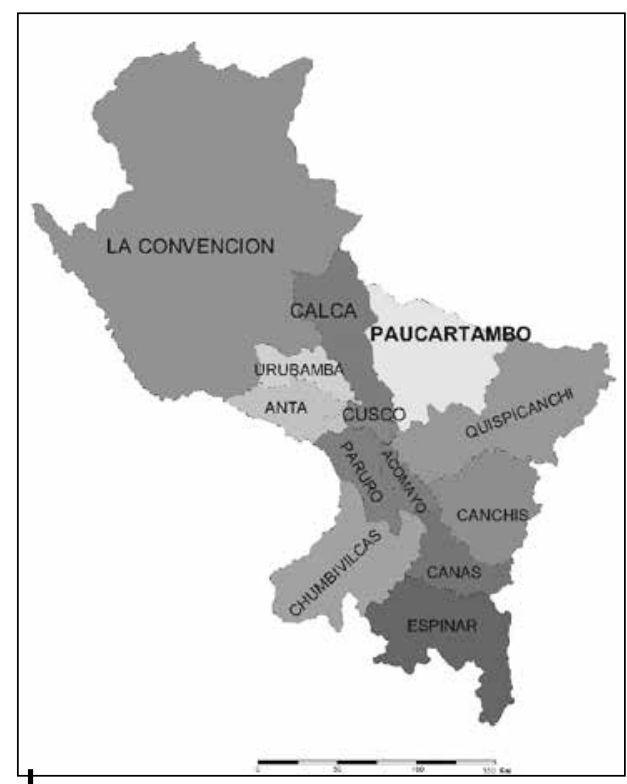

Figura 1 - Mapa del departamento del Cusco con la ubicación de la provincia de Paucartambo,

Elaborado a partir del mapa del Perú de Wikipedia Commons es que trajo en dos cajones el ajuar funerario que se encuentra en el museo y manifestó que lo cedía al museo insinuando que se le diera una gratificación. Pero el Consejo Universitario no obstante que había el deseo de otorgarle dicha gratificación denegó la petición, alegando de que el mencionado Carlos Rodríguez estaba enjuiciado y volvió a la cárcel por espacio de dos años por el delito de excavación clandestina.

Se anota para su constancia. Los documentos referentes a este caso deben obrar en los archivos del Patronato Departamental de Arqueología, siendo conservador del museo el Sr. Dr. Leónidas Cárdenas Yépez y Director el Dr. Luis A. Pardo.

Teniendo en cuenta lo que fue registrado en 1947 en el Catálogo Razonado del Instituto Arqueológico del Cusco, el ajuar funerario de la tumba de Paucartambo contenía un gran número de objetos elaborados en diversos materiales: en madera (chuas, keros, cucharitas, bateas), en cerámica (chuas, pocillos, cántaros, ollas, vasitos, aríbalos), materiales de pintura (potes con pigmentos ocres y rojos, paleta, estuche), en lana y otros tejidos (sandalias, bolsitas, sombreros), en lapislázuli (collares), en valva de concha, en trenzas de cabello y en metales. Los tres metales —oro, plata y cobre- estaban también representados en este ajuar (solo un objeto en el rol, un peine, es elaborado en plomo). Además de sonajas, de una cinta, de dos tupus y de un cuchillo en cobre así como de un disco en 
cobre dorado, el ajuar contenía varios objetos en plata y oro; se destacan los de plata por su número.

Efectivamente, mientras que solamente cuatro tupus, una estatuilla femenina, un prendedor y un vasito del ajuar son en oro, hay un total de veintiséis objetos en plata que se distribuyen como sigue: un accorasi (placa metálica), veinte tupus de tamaños diversos y cinco estatuillas antropomorfas (ninguna estatuilla zoomorfa fue inventariada). Pertenecen también al ajuar veinte fragmentos de tupus en plata, pero no se sabe a cuántos tupus corresponden.

Las estatuillas antropomorfas incaicas, elaboradas en metal y en valva de concha spondylus, con características propias relacionadas con su sexo, son a veces encontradas con sus indumentarias, tocados de plumas y vestimentas prendidos por cintas y tupus (por ejemplo Dransart, 1995; Benson, 2001; Bortoluzzi \& Martínez Armijo, 2011). Los tupus (que fueran también empleados en vida) son ornamentos metálicos de la vestimenta utilizados para prender los paños que envuelven la estatuilla (Cieza de León, 1922; Vetter, 2007; Fernández Murillo, 2015; Vetter, 2016).

Entre las estatuillas antropomorfas del ajuar de Paucartambo, de tamaños distintos y elaboradas por las técnicas del vaciado o por laminado, se destacan dos por su singularidad. Una es la figura femenina, la más grande del conjunto, elaborada con metales distintos, cabeza y busto en aleación de plata y piernas en aleación de cobre (fig. 2). A nuestro conocimiento, es la única estatuilla antropomorfa bicolor de proveniencia conocida. Se señala también la singularidad de la ofrenda intacta, constituida por una estatuilla femenina en su caja original de madera, envuelta en paños de lana prendidos por dos tupus de plata.

Además de los dos tupus que prenden los paños de la figura femenina de la ofrenda intacta, también figuran en el del ajuar de Paucartambo otros cinco tupus miniatura, sueltos. Las dimensiones y tipología de los siete tupus están muy próximas (fig. 3)2. Se podría así sospechar que los tupus sueltos pertenecerían inicialmente a las otras estatuillas de plata del hallazgo, pero su número (cinco) parece indicar un conjunto probablemente incompleto o de tupus o de estatuillas ${ }^{3}$.

Sin embargo, el conjunto de estatuillas antropomorfas de la tumba de Paucartambo (fig. 2) parece bastante heterogéneo desde el punto de vista tipológico y tecnológico. Las cinco estatuillas femeninas y una masculina tienen tamaños muy diversos, una es de oro y las otras son de plata, y fueron fabricadas, como se expone abajo, unas por la técnica del vaciado y otras por la técnica del laminado. Para ver hasta qué punto esta heterogeneidad se verifica, las estatuillas antropomorfas

2 Otros tupus miniatura de la región de Cusco elaborados en aleaciones de oro y de cobre tienen tipología análoga a la de nuestros tupus. Por ejemplo en la colección del Museo de la Casa Garcilaso en Cusco se puede encontrar un tupo de cerca de $5 \mathrm{~cm}$ en bronce arsenical excavado en Pikillacta (Lechtman \& Macfarlane, 2005).

3 Ninguno tupo miniatura en oro procedente de la tumba de Paucartambo fue registrado en el Catálogo Razonado del Museo Inka. 


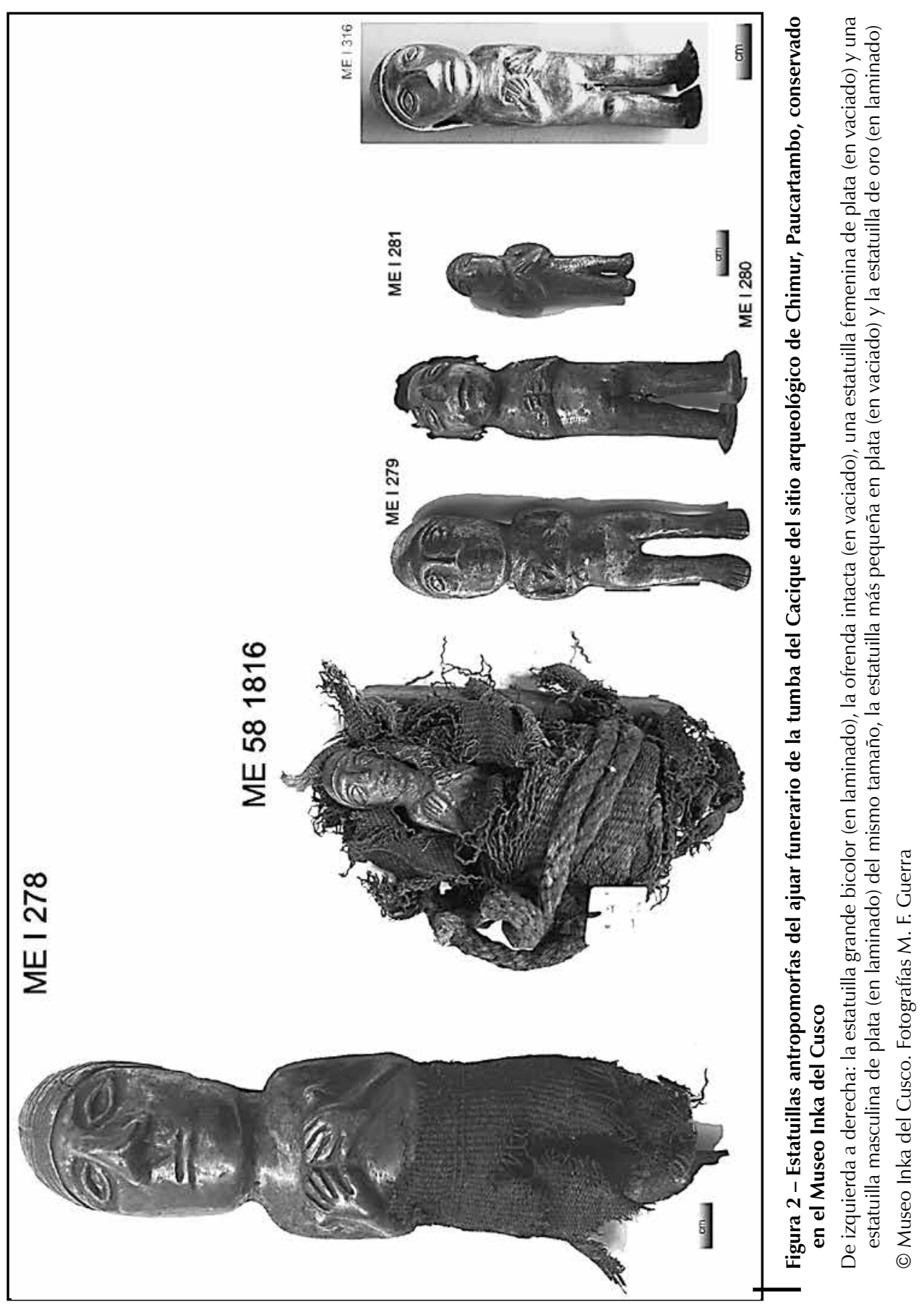




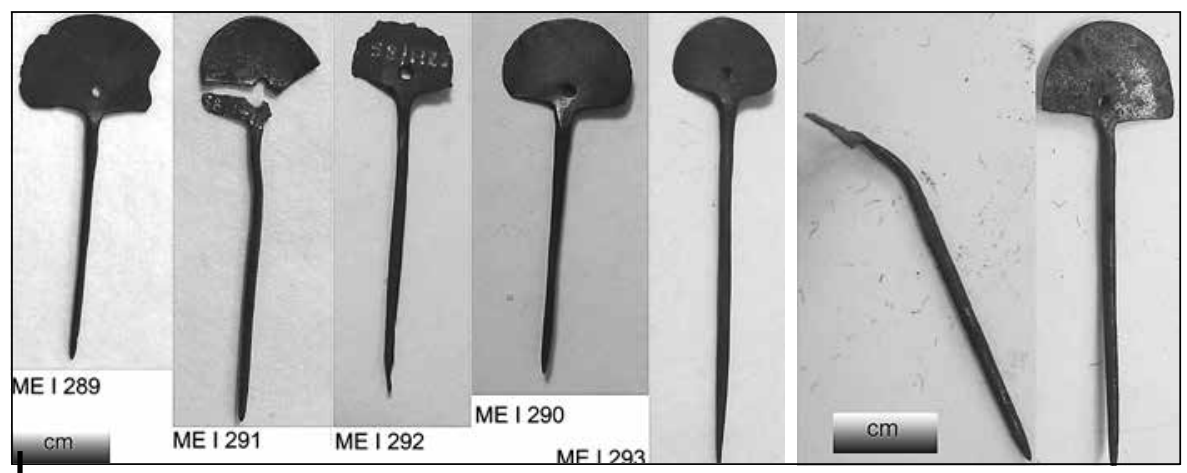

Figura 3 - Tupus miniatura en plata del ajuar funerario de la tumba del Cacique del sitio arqueológico de Chimur, Paucartambo, conservado en el Museo Inka del Cusco

De izquierda a derecha: los cinco tupus sueltos y los dos tupus de la estatuilla ME 581816

(C) Museo Inka del Cusco. Fotografías M. F. Guerra

y los tupus miniatura del hallazgo de Paucartambo fueron estudiados por técnicas físico-químicas no destructivas que proporcionan información sobre las prácticas de los orfebres y las aleaciones empleadas.

\section{METODOLOGÍA}

La oportunidad única conferida por la Dirección del Museo Inka nos permitió una primera aproximación a los procedimientos de los orfebres para la fabricación de las estatuillas y tupus miniatura en plata que constituyen las ofrendas incaicas en la región del Cusco a fin de entender la construcción de una estatuilla bicolor. El trabajo fue completado con el estudio de la única estatuilla antropomorfa en oro del ajuar.

Los objetos fueron estudiados mediante microscopía óptica y fluorescencia de rayos X (FRX) para determinar sus tecnologías de producción y las aleaciones utilizadas para su fabricación. Los equipos fueron transportados desde el laboratorio fisicoquímico de la Dirección Desconcentrada de Cultura Cusco hasta una sala del Museo Inka para proceder al estudio in situ.

El análisis micromorfológico fue efectuado con un estéreo microscopio marca Carl Zeiss, Modelo Discovery V8. Fueron sacadas imágenes con ayuda de una cámara Canon, equipada para macrofotografía y con un microscopio digital Pro de Celestron con sensor de 5MP. El análisis químico de las aleaciones fue efectuado con el equipo portátil Thermo Scientific Niton Modelo XL3T 950 GOLDD, en modo Metales Preciosos, con un haz colimado hasta $3 \mathrm{~mm}$ y $50 \mathrm{kV}$ de potencia. Se hicieron dos análisis para cada uno de los objetos elaborados por vaciado y tres a cinco para cada uno de los objetos elaborados por martillado.

El método no necesita de una preparación previa de la superficie (Guerra, 2008), pero los resultados obtenidos corresponden a la superficie del objeto, cerca de 
10-20 $\mu$ m según la aleación y el elemento determinado (Troalen et al., 2014). Para validar los resultados obtenidos por FRX y verificar la exactitud de nuestro método de análisis químico, se analizaron previamente padrones de plata de Goodfellow y padrones de oro de CLAL (Comptoir Lyon-Alemand, Louyot et Cie) elaborados con varias aleaciones. Los cuadros 1 y 2 muestran la buena concordancia de los resultados obtenidos con los valores de referencia.

Cuadro 1 - Comparación de la composición certificada (VC) y determinada por FRX (VD) para los padrones de oro $(\mathrm{Au}=$ oro; $\mathrm{Ag}=$ plata; $\mathrm{Cu}=$ cobre)

\begin{tabular}{|lcccc|}
\hline Referencia & & Au $\%$ & Ag $\%$ & Cu $\%$ \\
\hline Std 1 & VC & $\mathbf{9 3 , 1}$ & $\mathbf{3 , 0}$ & $\mathbf{3 , 9}$ \\
& VD & 92,9 & 3,8 & 3,3 \\
\multirow{3}{*}{ Std 2 } & VC & $\mathbf{9 0 , 3}$ & $\mathbf{0 , 3}$ & $\mathbf{9 , 4}$ \\
& VD & 90,6 & 0,3 & 9,1 \\
Std 3 & VC & $\mathbf{9 0 , 8}$ & $\mathbf{6 , 3}$ & $\mathbf{2 , 9}$ \\
& VD & 90,6 & 6,8 & 2,6 \\
Std 4 & VC & $\mathbf{9 3 , 6}$ & $\mathbf{6 , 1}$ & $\mathbf{0 , 3}$ \\
& VD & 93,1 & 6,7 & 0,3 \\
Std 6 & VC & $\mathbf{7 5 , 5}$ & $\mathbf{6 , 0}$ & $\mathbf{1 8 , 5}$ \\
& VD & 75,1 & 6,0 & 19,0 \\
\multirow{3}{*}{ Std 7 } & VC & $\mathbf{7 6 , 0}$ & $\mathbf{1 7 , 0}$ & $\mathbf{7 , 0}$ \\
& VD & 76,0 & 16,7 & 7,3 \\
\hline
\end{tabular}

Cuadro 2 - Comparación de la composición certificada (VC) y determinada por FRX (VD) para los padrones de plata (Ag=plata; $\mathrm{Cu}=$ cobre)

\begin{tabular}{|lccc|}
\hline Referencia & & Ag $\%$ & Cu \% \\
\hline Goodfellow 1 & VC & $\mathbf{9 5}$ & $\mathbf{5}$ \\
& VD & 95 & 5 \\
Goodfellow 2 & VC & $\mathbf{9 2 , 5}$ & $\mathbf{7 , 5}$ \\
& VD & 92,9 & 7,1 \\
Goodfellow 3 & VC & $\mathbf{8 0}$ & $\mathbf{2 0}$ \\
& VD & 81 & 19 \\
& VC & $\mathbf{7 1}$ & $\mathbf{2 9}$ \\
& VD & 69 & 31 \\
\hline
\end{tabular}




\section{LOS TUPUS}

En el conjunto de tupus hallados en la tumba de Paucartambo se cuentan dos de cobre, cuatro de oro y veintiséis de plata, más otros veinte fragmentos de tupus de plata. Entre los tupus de plata no fragmentados, siete son miniaturas, dos de los cuales, como referido, pertenecen a la ofrenda intacta y se encuentran todavía sobre la indumentaria de la estatuilla femenina, dentro de su caja de madera. Uno de estos dos tupus está muy degradado, se supone debido al contenido de humedad de la atmosfera ambiental, al contacto con los textiles de lana, y posiblemente con la madera (Watkinson, 2010; Hodgkins et al., 2013; Guerra \& Tissot, 2013; Tissot et al., 2016). También, los tejidos se encuentran muy degradados, pero todavía se puede ver en las fibras algunas partes rojas y otras posiblemente amarillas, como se espera en la indumentaria de las estatuillas incaicas (por ejemplo Mignone, 2015; Arnold \& Dransart [eds.], 2014).

Las dimensiones de los siete tupus miniatura, de cabeza semicircular, son similares, así como su forma (fig. 3); todos son elaborados en una sola pieza por las técnicas del vaciado y martillado (como relatado en Vetter, 2007), y todos presentan un orificio hecho por perforación, por donde pasaría un cordón de unión con su par, hoy inexistente. De entre los tupus del hallazgo de Paucartambo, uno de mayores dimensiones fue encontrado con los hilos de un tejido incaico en su orificio pasador. Debemos comentar que, por ejemplo los dos tupus miniatura de plata de la estatuilla femenina en plata de la ofrenda del cerro Gallán (perteneciente a la colección del Museo Etnográfico Juan B. Ambrosetti, Buenos Aires) tienen todavía el cordón de unión (Guardia \& Rojas, 2013).

La punta de los tupus es afilada para poder entrar en los tejidos, como se muestra en la figura 4. El espesor de la placa de plata, la forma de perforar los orificios más el acabado en los tupus son distintos, lo que cohíbe la posibilidad de que hayan sido hechos por el mismo platero. La figura 5 muestra los orificios de cinco de los siete tupus estudiados (dos están fragmentados, lo que hace imposible ver el orificio completo). El tupu ME I 289 es el único que presenta todavía la plata restante de la perforación del orificio, y la perforación del tupu ME I 293 podría haber sido hecha (por lo menos inicialmente) en el molde.

Los tupus presentan estados de preservación muy distintos, algunos se encuentran muy degradados, en particular ME I 291, ME I 292 y uno de los tupus de la estatuilla ME 58 1816. Su análisis químico muestra todavía que sus composiciones (cuadro 3) son análogas. Solamente uno de los tupus, el de referencia ME I 289,

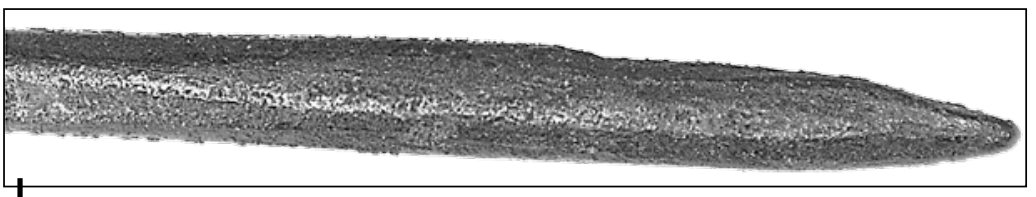

Figura 4 - Detalle de la punta del tupu ME I 290

(C) Museo Inka del Cusco. Fotografía M. F. Guerra 

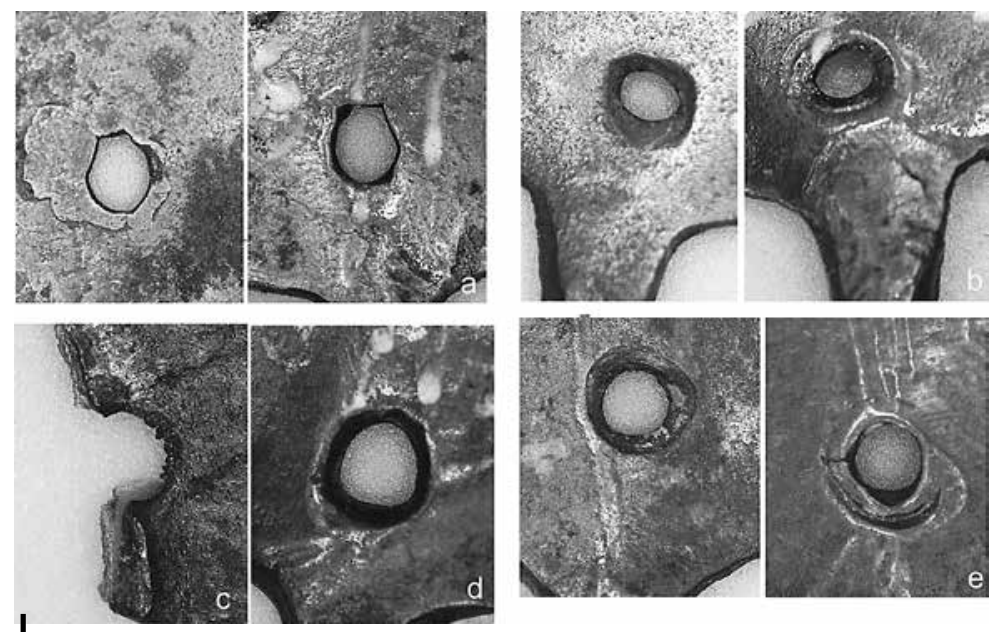

Figura 5 - Detalle de los orificios de cinco tupus miniatura en plata intactos del ajuar funerario de la tumba de Paucartambo: (a) los dos lados del ME I 289, uno de los lados presenta la plata restante de la perforación; (b) los dos lados del ME I 290 donde se ve el sentido de la perforación; (c) uno de los lados (iguales) de uno de los tupus de la estatuilla ME 581816 (el otro tupu está incompleto); (d) uno de los lados (iguales) del ME I 292; (e) los dos lados del ME I 293 donde se ve el sentido de la perforación

(C) Museo Inka del Cusco. Fotografías M. F. Guerra

es de mejor calidad, pues contiene apenas 3\% de cobre en vez de 5-6\% como los otros. Como se muestra en el cuadro 3, los dos tupus de la ofrenda intacta tienen una composición química similar; podrían así haber estado elaborados con la misma aleación y por el mismo platero.

Cuadro 3 - Composición de los tupus de plata miniatura del ajuar funerario de la tumba de Paucartambo obtenida por FRX ( $\mathrm{Au}=$ oro; $\mathrm{Ag}=$ plata; $\mathrm{Cu}=$ cobre; $\mathrm{Pb}=$ plomo; $\mathrm{Sn}=$ estaño)

\begin{tabular}{|lccccc|}
\hline Referencia & $\mathbf{A g} \%$ & $\mathbf{C u} \%$ & $\mathbf{A u} \%$ & $\mathbf{P b} \%$ & Sn \% \\
\hline ME 58 1816 - tupo 1 & 93,7 & 5,1 & 0,4 & 0,3 & 0,5 \\
& 93,6 & 5,6 & 0,5 & 0,3 & \\
ME 58 1816 - tupo 2 & 93,0 & 5,4 & 0,5 & 0,2 & 0,9 \\
ME-I 290 & 93,9 & 6,0 & 0,1 & 0,1 & \\
ME-I 292 & 92,7 & 6,2 & 0,2 & 0,9 & \\
ME-I 292 & 92,7 & 6,2 & 0,2 & 0,9 & \\
ME-I 291 & 93,2 & 6,1 & 0,4 & 0,2 & \\
ME-I 289 & 96,4 & 3,0 & 0,4 & 0,2 & \\
\hline
\end{tabular}


En todos los tupus se encontraron pequeñas cantidades de oro y de plomo en la aleación de plata. El oro es normalmente característico del mineral de plata y el plomo puede venir del tratamiento metalúrgico del mineral de plata, del procedimiento de refinado (por ejemplo Petersen G., 1970; Zori \& Tropper, 2010).

\section{LAS ESTATUILLAS}

El conjunto de estatuillas del hallazgo de Paucartambo cuenta con una estatuilla femenina en oro y cinco estatuillas antropomorfas de plata, una masculina y cuatro femeninas, de dimensiones muy distintas y elaboradas con técnicas también diferentes (fig. 2). La estatuilla masculina fue elaborada por martillado y repujado, mientras que las estatuillas femeninas, con excepción de la grande bicolor y la de oro, fueron elaboradas por vaciado.

\section{1. Detalles de elaboración}

\section{1. 1. Estatuillas laminadas}

Las estatuillas laminadas son típicas del trabajo del orfebre inka, como es descrito por ejemplo por Rowe (1996). Los distintos elementos que las forman fueron producidos en láminas repujadas, enroladas y después soldadas. Se encontraron vestigios de soldadura de las láminas de plata y de oro en diversas partes de las estatuillas, como se muestra en la figura 6. Las piernas en aleación de cobre de la estatuilla bicolor también fueron elaboradas por laminado. Se supone que sus pies, hoy inexistentes, debieron ser elaborados en aleación de cobre por laminado.

La estatuilla masculina fue soldada entre las piernas, detrás del torso y de la cabeza. Sus pies y sus orejas fueron hechos separadamente y luego soldados, al contrario del miembro masculino y del llautu alrededor de la cabeza; los dos fueron elaborados en repujado directamente en la lámina de plata ${ }^{4}$. El tupu de la cabeza es todavía una pieza distinta que termina el objeto. La estatuilla femenina en oro fue soldada también entre las piernas y detrás del torso. Sus pies y su cabello fueron hechos separadamente y luego soldados. Las láminas de plata fueron pulidas y los dedos formados por la técnica del cincelado (fig. 7).

La parte en plata de la estatuilla femenina bicolor que constituye la cabeza y el busto fue realizada de la misma manera de modo tradicional, el cabello fue elaborado por separado y después soldado a la cabeza y torso. Las dos partes que forman esta estatuilla (cabeza y tronco en plata y piernas en cobre) no fueran soldadas, solamente encajadas (fig. 8). Las piernas están cubiertas con un textil

4 Normalmente el llautu es elaborado en dos partes soldadas a la cabeza y el miembro masculino es fabricado a partir de una hoja de plata enrollada y soldada y después encajada y soldada al torso. La figura masculina del ajuar de Paucartambo parece realizada de otro modo. Su miembro masculino podría haber sido elaborado por repujado y soldado pero, debido a la degradación de la superficie, es imposible verificar la existencia de soldadura. 

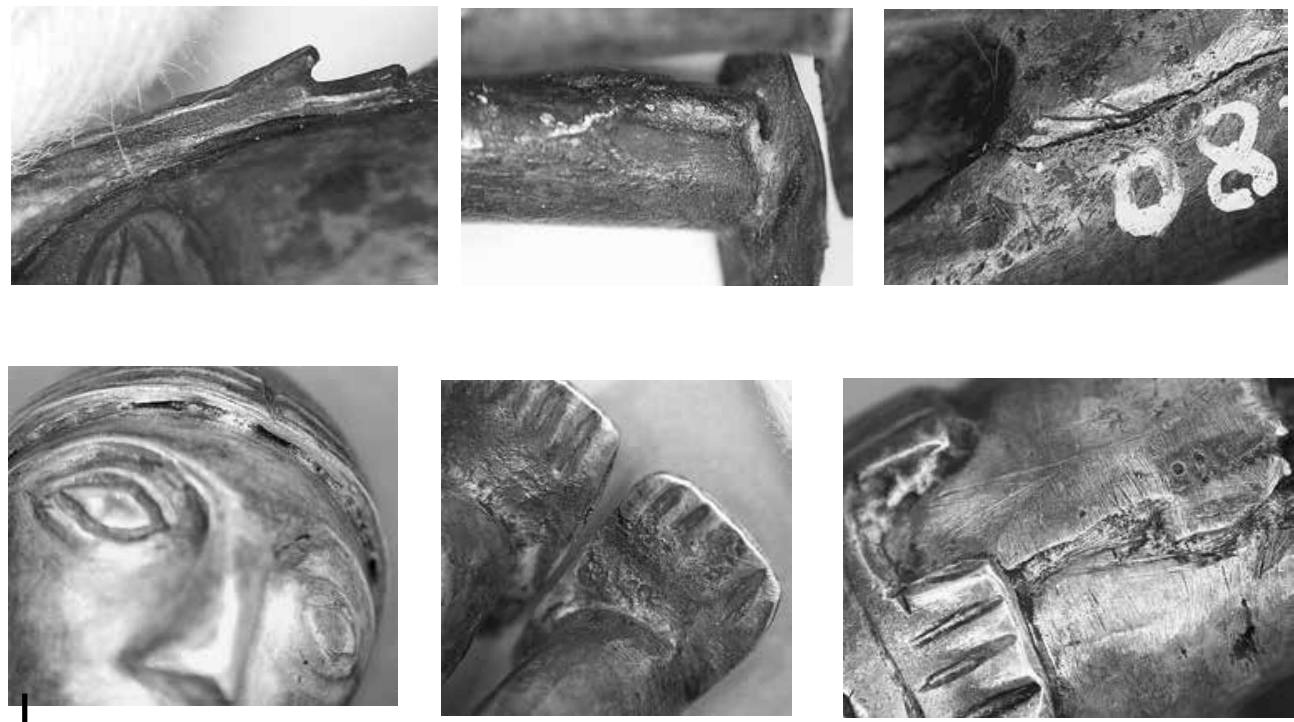

Figura 6 - Detalles de las regiones de unión por soldadura de las diversas láminas martilladas y repujadas de la estatuilla masculina en plata ME I 280 (arriba) y de la estatuilla femenina en oro ME I 316 (abajo)

(C) Museo Inka del Cusco. Fotografías M. F. Guerra
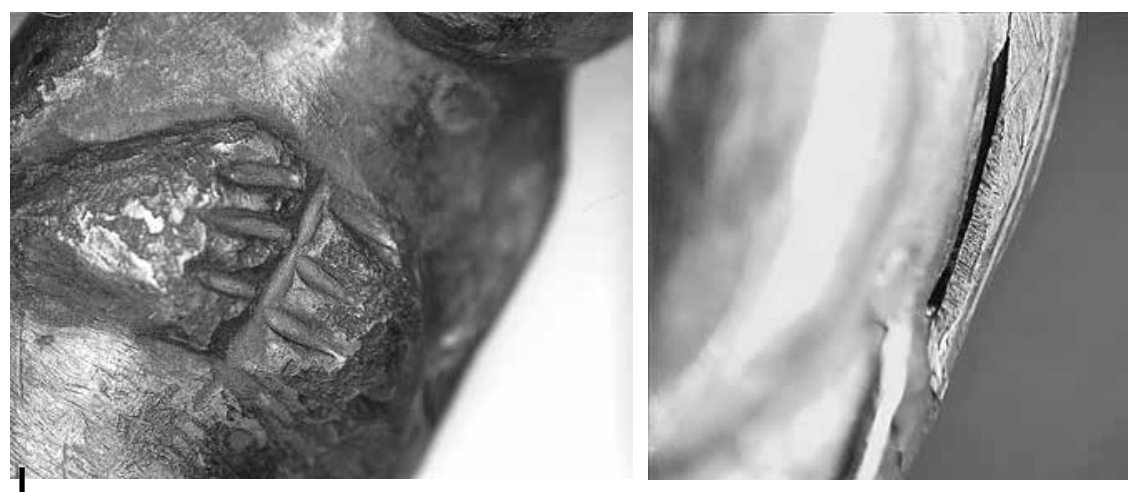

Figura 7 - Los dedos en cincelado de la estatuilla masculina en plata ME I 280 (izquierda) y la lámina en oro pulida del cabello de la estatuilla femenina en oro ME I 316 (derecha)

(C) Museo Inka del Cusco. Fotografías M. F. Guerra

de lana, que originalmente debió ser colorido, pues se encontraron vestigios microscópicos de colores rojo y amarillo (Phipps, 2015). El contacto con la lana ciertamente aceleró el ratio de la corrosión atmosférica de las piernas en cobre que se encuentran muy degradadas. 

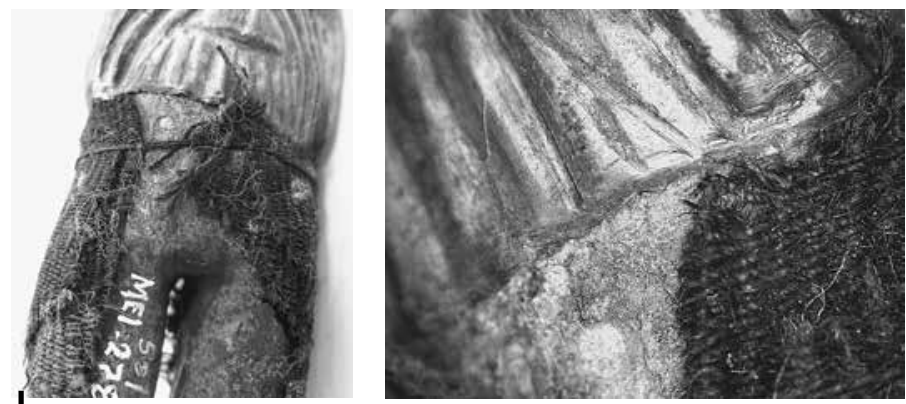

Figura 8 - Detalle del encaje sin soldadura de las dos partes que constituyen la estatuilla femenina bicolor ME I 278

(C) Museo Inka del Cusco. Fotografías M. F. Guerra

\section{1. 2. Estatuillas vaciadas}

Las otras estatuillas femeninas fueran realizadas con la técnica de vaciado en molde, luego pulidas y a veces acabadas, en general por cincelado, en particular las manos, los pies y el cabello. El acabado no es meticuloso: por ejemplo en ciertas regiones de las estatuillas ME I 279 y ME I 281 el metal de exceso no fue retirado y la superficie no fue pulida, como entre las piernas, alrededor del cabello y en el cuello. Los pies de la segunda estatuilla no tienen dedos y los dedos de las manos fueron solamente dibujados en el molde. A pesar de ser elaboradas con técnicas idénticas y con una calidad análoga de acabado, el trabajo no fue ejecutado por el mismo platero, como evidencian los detalles de la figura 9.

Por su estado delicado, no fue posible separar la estatuilla femenina ME 581816 de sus textiles de lana. Los tejidos están adheridos a los productos de corrosión formados en su superficie, pero el vaciado y el acabado parecen más minuciosos.

La única que parece haber tenido un problema con la temperatura del metal durante la fundición es la estatuilla ME I 281, pues tiene espacios vacíos de metal en las piernas y en los brazos, como se muestra en la figura 10.

\section{2. Detalles tipológicos}

Todas las estatuillas femeninas estudiadas tienen los atributos típicos de las estatuillas incaicas: el peinado tradicional, el sexo, los senos, los brazos doblados sobre el pecho, y la protuberancia de la bola de hojas de coca. La estatuilla masculina tiene también los atributos típicos: los brazos doblados sobre el pecho, la protuberancia de la bola de hojas de coca, el miembro masculino erecto, los largos lóbulos de las orejas perforados, y el llautu alrededor de la cabeza.

No tenemos con que comparar la estatuilla masculina en el conjunto de Paucartambo, pero podemos concentrarnos en los detalles de las estatuillas femeninas. Pese a la semejanza de los atributos, las posturas (por ejemplo la forma como la cabeza entra en la espalda) y las deformaciones craneanas de las 

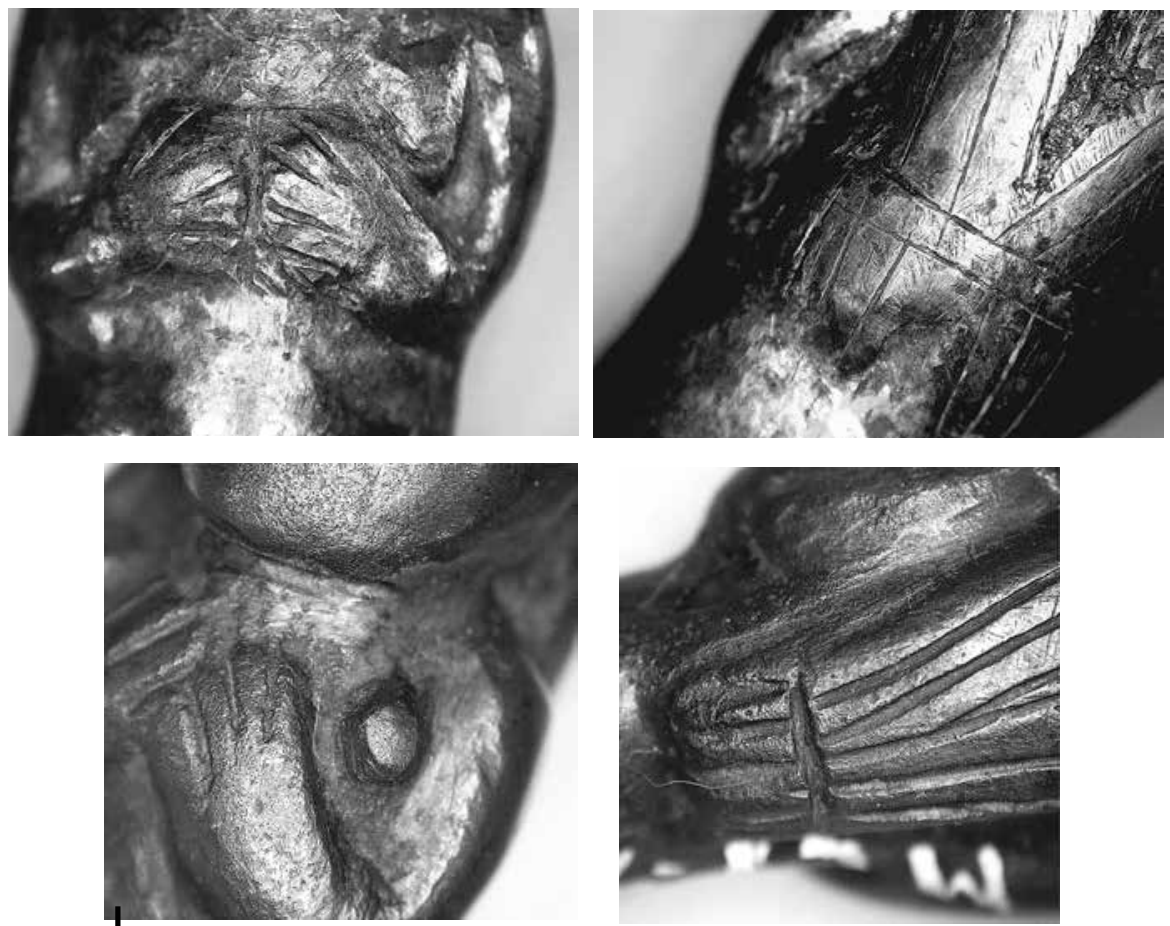

Figura 9 - Detalles de las manos y del cabello de las estatuillas femeninas en plata elaboradas por vaciado ME I 279 (arriba) y ME I 281 (abajo)

(C) Museo Inka del Cusco. Fotografías M. F. Guerra

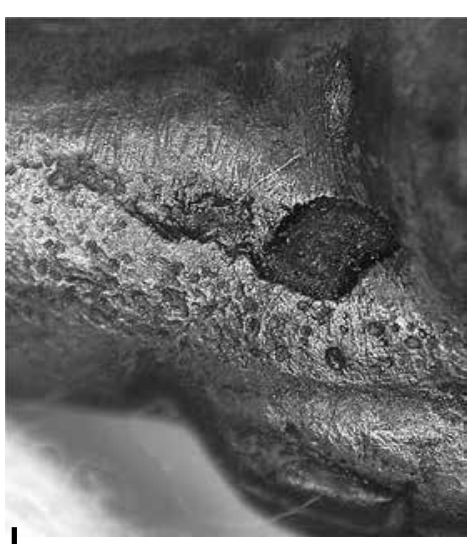

Figura 10 - Detalle de la falta de metal en las piernas de la estatuilla femenina en plata ME I 281 elaborada por vaciado

(C) Museo Inka del Cusco. Fotografías M. F. Guerra cinco estatuillas femeninas (esta deformación fue también detectada en las momias capacochas, como se describe por ejemplo en Ceruti, 2003) son distintas y parecen indicar un trabajo hecho por varios orfebres. El segundo detalle más notable es el peinado, como se muestra en la figura 11; es siempre con partidura o raya al medio llegando hasta la cintura en el dorso (como es usual en las momias precolombinas, Arriaza et al., 1986). El cabello del peinado en las estatuillas ME I 279 y ME I 316 termina separado en dos en el dorso, al contrario del peinado de las otras tres estatuillas. Pero es el peinado de la estatuilla bicolor que se diferencia más, pues la marca de partidura al medio es más profunda, tal como en la estatuilla ME I 279. Encima de su cabello fue colocado un adorno que no corresponde al peinado tradicional de las estatuillas incaicas. Se señala el peinado de la estatuilla de plata laminada V A 28917 del Museo de Etnología de Berlín descrita en Guerra et al., en ese mismo número. Con una profunda marca de 


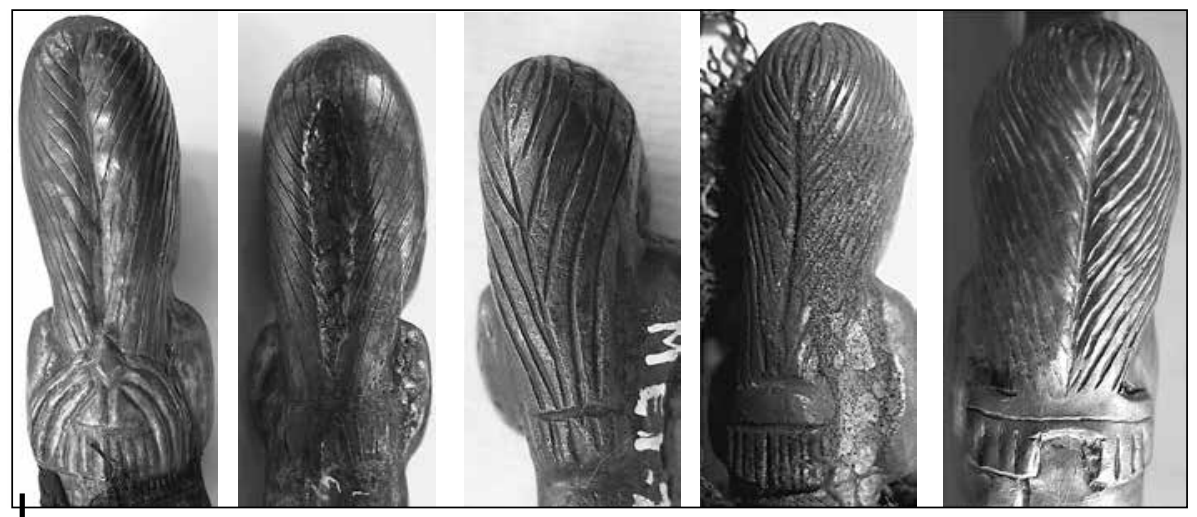

Figura 11 - Los peinados de las estatuillas femeninas, de izquierda a derecha, ME I 278 (bicolor, elaborada por martillado y repujado), ME I 279 (elaborada por vaciado), ME I 281 (elaborada por vaciado), ME 581816 (de la ofrenda intacta, elaborada por vaciado), ME I 316 (elaborada por martillado y repujado)

(C) Museo Inka del Cusco. Fotografías M. F. Guerra

partidura al medio, su peinado también termina de una forma poco tradicional, con pequeñas trenzas y al parecer un adorno adicional. Se podría conjeturar que, siendo los peinados de estas estatuillas menos tradicionales, se trataría de producciones del periodo de contacto (¿o colonial?) $)^{5}$.

\section{3. Aleaciones empleadas}

Las aleaciones utilizadas en la elaboración de las estatuillas antropomorfas provenientes del hallazgo de Paucartambo fueron estudiadas por FRX. Para las estatuillas vaciadas se hicieron dos a tres puntos de análisis. Para las otras, siempre que fue posible, fueron realizadas análisis de las distintas láminas que las constituyen. Los resultados obtenidos se encuentran en el cuadro 4.

La estatuilla bicolor ME-I 278 fue elaborada con una aleación de plata de muy buena calidad y con un bronce conteniendo muy poco estaño. La estatuilla masculina ME-I 280, también elaborada por martillado y repujado, es de muy buena calidad. Se señala que los pies (sin embargo originales) son hechos con una aleación conteniendo un poco más de cobre, distinta de la del cuerpo.

Dos de las tres estatuillas elaboradas por vaciado (una es la estatuilla de la ofrenda intacta) tienen una composición análoga, pero distinta a la composición de las estatuillas elaboradas por martillado, mientras que la tercera, ME I 281, tiene una composición equivalente a las estatuillas elaboradas por martillado.

5 M. E. Gentile ubica la capacocha del Cerro Aconcagua «como la más cercana en el tiempo a la presencia hispana» pero las dos estatuillas antropomorfas son masculinas y así no es posible comparar el peinado con los de las estatuillas de Paucartambo (Gentile, 2010). 
Cuadro 4 - Composición de las estatuillas antropomorfas de plata y oro del ajuar funerario de la tumba de Paucartambo obtenida por FRX

\begin{tabular}{|llccccc|}
\hline Referencia & & Ag $\%$ & Cu \% & Au \% & Pb \% & Sn \% \\
\hline ME I 278 & piernas & 0,2 & 99,8 & & & 2,4 \\
ME I 278 & cabeza y busto & 98,0 & 1,8 & 0,1 & 0,1 & \\
& & & & & & \\
ME I 280 & cuerpo & 97,3 & 1,6 & 0,9 & 0,1 & \\
ME I 280 & pies & 96,1 & 3,4 & 0,4 & 0,1 & \\
& & & & & & \\
ME I 279 & cuerpo & 78,4 & 19,0 & 2,4 & 0,3 & \\
ME I 281 & cuerpo & 96,0 & 3,5 & 0,3 & 0,2 & \\
ME 58 1816 & cuerpo & 86,0 & 13,3 & 0,5 & 0,2 & \\
& & & & & & \\
ME I 316 & cuerpo y cabello & 51,2 & 3,2 & 45,6 & & \\
ME I 316 & dorso con suelda & 51,4 & 5,1 & 43,5 & & \\
\hline
\end{tabular}

La estatuilla en oro ME I 316 es realizada en una aleación que contiene cerca de $45 \%$ de plata. Fue posible efectuar el análisis de la región del dorso donde el cabello fue soldado al cuerpo (ver fig. 6). Como se esperaba, en esta región la concentración de cobre es más elevada. El área de análisis del haz de rayos X es demasiado ancho para que solo la soldadura sea analizada; la composición obtenida, siendo la media de la composición de la soldadura y de la hoja de oro, muestra que la soldadura fue elaborada con una aleación también de oro y plata pero conteniendo valores de cobre más elevados para bajar el punto de fusión.

En la figura 12 se compara la composición de las estatuillas con los siete tupus, incluidos los de la ofrenda. Exceptuando uno de los tupus, estos objetos forman un grupo homogéneo distinto de las estatuillas. El tupu que se diferencia del grupo es parecido a la estatuilla ME I 281; esta última se distingue de las otras estatuillas elaboradas por vaciado. Esta estatuilla, elaborada por vaciado, contiene efectivamente más cobre. Se podría suponer que el tupu y la estatuilla formaban originalmente un conjunto.

Al contrario de los tupus, las estatuillas se separan en dos grupos (entre los dos se encuentra el grupo de los tupus). La temperatura de fundición de las aleaciones del grupo conteniendo más cobre alcanza casi $825^{\circ} \mathrm{C}$ y casi $950^{\circ} \mathrm{C}$ para el otro grupo 6. No obstante, es imposible relacionar estas aleaciones con la tecnología específica utilizada por los plateros (laminado o vaciado), pero las figuras laminadas fueran producidas con una aleación de mejor calidad.

A nuestro conocimiento, solamente se conoce la composición de una estatuilla antropomorfa de plata de la región de Cusco. Esta figura femenina, procedente del

6 La temperatura de fundición de las piernas en bronze de la estatuilla es cerca de $1080^{\circ} \mathrm{C}$. 


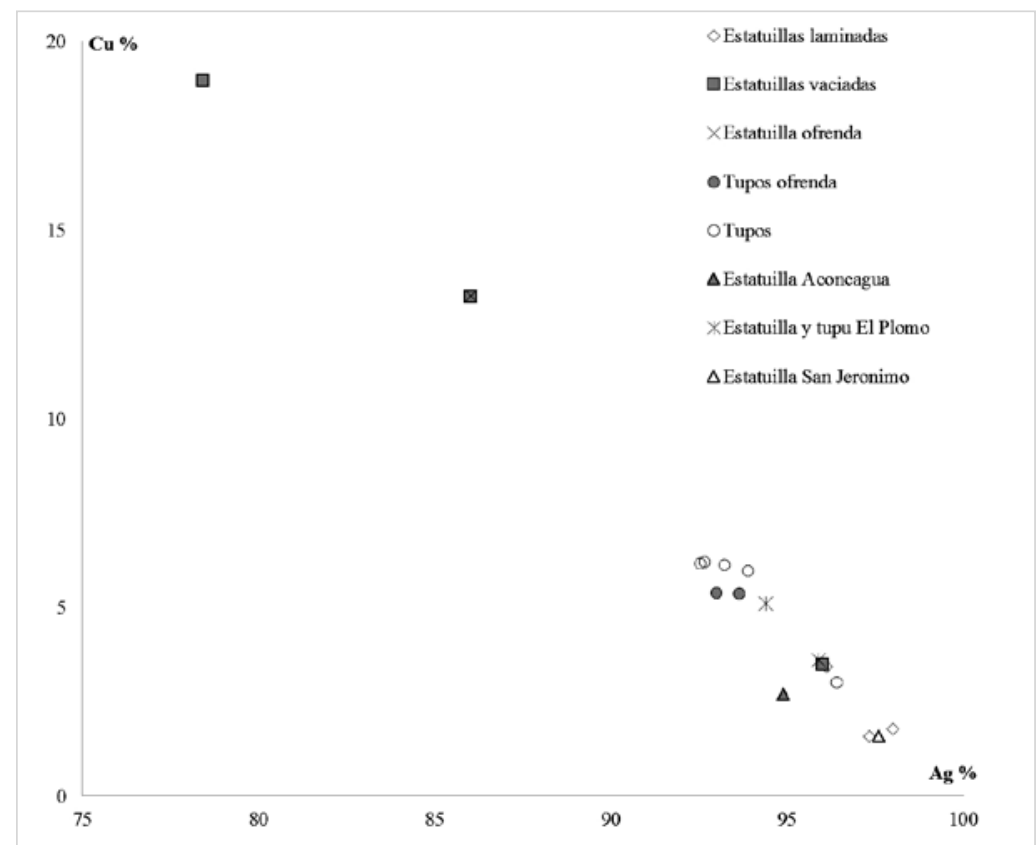

Figura 12 - Comparación de las concentraciones de plata (Ag \%) y cobre (Cu \%) determinadas por FRX para los tupus y las estatuillas del hallazgo de Paucartambo y valores publicados para las estatuillas y tupos de San Jerónimo (Rovira Llórens y Gómez Ramos, 1995), Cerro Aconcagua (Bárcena, 2004) y Cerro El Plomo (Oberhauser et al., 1059). Elaborado por J. C. S. Palminos \& M. F. Guerra

distrito de San Jerónimo, fue ejecutada en martillado y pertenece a la colección Larrea del Museo de América en Madrid (referencia MA 07046). Su análisis, publicado por Rovira Llórens \& Gómez Ramos (1995), indica 97,6 \% de plata y $1,6 \%$ de cobre, lo que corresponde exactamente a la composición de nuestras estatuillas ME I 278 y ME I 280, igualmente elaboradas por martillado.

Fueron también hasta hoy analizados los objetos metálicos de dos ajuares funerarios de momias capacocha: los de la momia del Cerro Aconcagua en Argentina y los de la momia del Cerro El Plomo en Chile. Los dos ajuares contienen estatuillas en oro y plata.

La estatuilla antropomorfa en plata del ajuar funerario de la momia del Cerro Aconcagua fue elaborada con la técnica de laminado y su composición, publicada por Bárcena en 2004 (94,9\% Ag, 2,7\% Cu, 1,6\% Au), se acerca a nuestro grupo de estatuillas laminadas de mejor calidad. La estatuilla femenina laminada del ajuar de la momia del Cerro El Plomo, cuya composición fue publicada en 1957-1959 por Oberhauser et al. (94,4 \% Ag, 5,1 \% Cu, 0,4\% Au), fue también elaborada con el mismo tipo de aleación.

En cuanto a la única estatuilla en oro del ajuar funerario de la tumba de Paucartambo, ME I 316, se puede comparar su composición con las raras estatuillas publicadas 
hasta hoy. Se encuentra en la misma colección de Madrid una estatuilla zoomorfa en oro (MA 07404) que también proviene del distrito de San Jerónimo y cuya composición (53,3\% Au, 42,4\% Ag y 4,3\% Cu, Rovira Llórens \& Gómez Ramos, 1995) es muy análoga a la de nuestra estatuilla en oro. Del mismo modo, la lámina y la plaquita de oro del ajuar funerario de la momia del Cerro Aconcagua tienen composiciones idénticas a nuestra estatuilla de oro (lámina 52,5\% Au, 43,9 $\mathrm{Ag} \%, 4,0 \% \mathrm{Cu}$ y plaquita $51,4 \% \mathrm{Au}, 45,8 \% \mathrm{Ag}, 3,6 \% \mathrm{Cu}$ ), pero las estatuillas antropomorfa y zoomorfa en oro de este mismo ajuar, elaboradas por laminado con aleaciones conteniendo altos valores de plata, muestran aleaciones distintas de las nuestras, que solamente contienen, respectivamente, $21 \%$ y $27 \%$ de plata ${ }^{7}$ (Bárcena, 2004). El ajuar de la momia del Cerro El Plomo contenía solamente una figura zoomorfa en oro. Su composición $(37,8 \% \mathrm{Au}, 43,1 \% \mathrm{Ag}, 19,1 \% \mathrm{Cu}$, Oberhauser et al., 1957-1959) muestra una concentración de plata próxima de nuestra estatuilla, pero su concentración de cobre es mucho más alta.

\section{CONCLUSIÓN}

El estudio de las seis estatuillas antropomorfas y de los siete tupus miniatura, encontrados en la tumba de Cacique del sitio arqueológico de Chimur, en Paucartambo, fue una oportunidad única que nos fue proporcionada por la Dirección del Museo Inka del Cusco para determinar los procedimientos de los orfebres en la elaboración de los objetos de las ofrendas incaicas en la región de Cusco, centro de la cultura Inka, incluyendo la construcción de una de las raras estatuillas bicolores conocidas.

Las técnicas de producción empleadas son el vaciado y laminado. En el último proceso, las láminas fueran martilladas, repujadas, soldadas y en su mayoría cinceladas. Además, las orejas de la estatuilla masculina son hechas a partir de hilos de sección cuadrada. Se diferencia la estatuilla bicolor cuyas dos partes, una en aleación de plata y la otra en aleación de cobre, no son soldadas pero solamente encajadas.

La tipología y las dimensiones de los tupus son bastante homogéneas, incluido el par de la indumentaria de la estatuilla de la ofrenda intacta. Sus composiciones son de forma idéntica muy homogéneas; solo uno es ligeramente de mejor calidad. Podríamos casi imaginar una producción regular y bien controlada en la capital del Imperio inka, con temperaturas de fundición bien determinadas y procedimientos regulares de afinación de metales. Sin embargo, las concentraciones de plomo son en general más elevadas en las aleaciones de los tupus, lo que podría indicar el empleo de plata que pasó por menos etapas del procedimiento metalúrgico (menos refinación). No hay razón de sospechar de la existencia de aprovisionamientos según los talleres de orfebres, pues deberían ser los mismos para la elaboración de las distintas tipologías de objetos.

7 Ver nota 5. Una diferencia de cronología podría explicar la diferencia de aleaciones utilizadas. 
Resalta la diversidad de las estatuillas antropomorfas del hallazgo. Esta diversidad se observa en la tecnología de producción empleada (vaciado y laminado), como también en el acabado y en algunos detalles tipológicos de las estatuillas como sus dimensiones, sus posturas, la forma de sus cráneos y los peinados. Otros detalles menos relevantes tales los ojos, la boca, etc. son también distintos, corroborando su diversidad y una elaboración por distintos plateros.

El conjunto cuenta solamente con una estatuilla de oro, cuya composición, por comparación con los raros objetos publicados del mismo tipo, aparece como incaica. La composición de las aleaciones empleadas en la elaboración de las cinco estatuillas de plata las separa en dos grupos, pero nada indica que hay una distinción por período de elaboración o por tecnología empleada. Uno de los tupus aparece como de composición idéntica a una de las estatuillas elaboradas por vaciado y podría así originalmente pertenecer a su posible indumentaria.

Los grupos composicionales obtenidos para las estatuillas de plata son distintos del grupo de los tupus, pero las estatuillas elaboradas en láminas de plata de proveniencia conocida (San Jerónimo y ajuares de las momias del Cerro Aconcagua y del Cerro El Plomo) se ubican en nuestro grupo de estatuillas laminadas.

En cuanto a la estatuilla bicolor, su peinado no es tradicional y sus dos colores son atípicos. Elaborada por laminado con una aleación de plata cuya composición es distinta de la de las otras estatuillas laminadas de Paucartambo y de la de San Jerónimo, se podría imaginar (pero nunca asegurar) una elaboración más reciente, posiblemente en el periodo de contacto (¿o colonial?).

Los datos tecnológicos obtenidos son insuficientes para avanzar hipótesis sobre la cronología del hallazgo de Paucartambo. Junto con la falta de información sobre el contexto de descubrimiento, estos datos abren diversas cuestiones sobre la proveniencia de los objetos inventariados en 1947. Se podría casi dudar que se trate del ajuar de una única tumba de Cacique. Pero sin embargo, los resultados obtenidos y su comparación con los otros resultados publicados invitan al desarrollo de mayores estudios físico-químicos de las producciones ceremoniales incaicas para obtener más datos sobre la metalurgia del oro y de la plata del Horizonte Tardío.

\section{Referencias citadas}

ARNOLD, D. \& DRANSART, P. (eds.), 2014 - Textiles, technical practice, and power in the Andes, 351 pp.; London: Archetype.

ARRIAZA, B. T., ALLISON, M. J., STANDEN, V., FOCACCI, G. \& CHACAMA, J., 1986 Peinados precolombinos en momias de Arica. Chungara: Revista de Antropología Chilena, 16-17: 353-375.

BÁRCENA, J. R., 2004 - Las piezas metálicas de la ofrenda ritual del Cerro Aconcagua. Mendoza, República Argentina. In: Tecnología del oro antiguo: Europa y América (A. Perea, I. Montero Ruiz \& Ó. García Vuelta, eds.): 157-172; Madrid: Anejos 
de Anejos de Archivo Español de Arqueología XXXII, Consejo Superior de Investigaciones Científicas.

BENSON, E. P., 2001 - Why Sacrifice? In: Ritual sacrifice in ancient Peru (E. P. Benson \& A. G. Cook, eds.): 1-33; Austin: University of Texas Press.

BORTOLUZZI, M. \& MARTíNEZ ARMIJO, I., 2011 - La muerte es el mensaje. La doble comunicación de la capacocha inca entre don y sacrificio. Thule. Rivista italiana di studi americanistici, 30/31: 208-228.

CATÁlOGO RAZONADO, 1947 - Museo e Instituto Arqueológico (hoy Museo Inka), Universidad Nacional San Antonio Abad Cusco.

CERUTI, M. C., 2003 - Elegidos de los dioses: identidad y estatus en las víctimas sacrificiales del volcán Llullaillaco. Boletín de Arqueología PUCP, 7: 263-275.

CIEZA DE LEÓN, P., 1922 - La crónica del Perú, 367 pp.; Madrid: Espasa-Calpe S.A.

DRANSART, P., 1995 - Elemental meanings: Symbolic expression in Inka miniature figurines, 58 pp.; Londres: Institute of Latin American Studies, University of London, Research Paper 40.

FERNÁNDEZ MURILLO, M. S., 2015 - Prendedores, topos y mujeres, 204 pp; La Paz: Museo Nacional de Etnografía y Folklore-MUSEF.

GENTILE, M., 2010 - Notas para una cronología relativa de las capacochas al sur de Charcas. In: Actas del XVII Congreso Nacional de Arqueología Argentina (J. R. Bárcena \& H. Chiavazza, eds.): 819-824; Cuyo: Departamento Científico de Arqueología, Universidad Nacional de Cuyo, tomo 2.

GUARDIA, S. H. de la \& ROJAS Z., A. M., 2013 - Carácter representacional de ofrendas textiles incaicas envíos para el más allá. Revista Diseña, 6, http://www.revistadisena. com/pdf/revistadisena_6_caracter-representacional-de-ofrendas-textiles.pdf

GUERRA, M. F., 2008 - An overview on the ancient goldsmith's skill and the circulation of gold in the past: the role of X-ray based techniques. X-ray Spectrometry, 37 (4): 317-327.

GUERRA, M. F. \& TISSOT, I., 2013 - The role of nuclear microprobes in the study and conservation of gold and silver cultural heritage items: an overview. Nuclear Instruments and Methods in Physics Research Section B, 306: 227-23.

HODGKINS, R. E., CENTENO, S. A., BAMBERGER, J. A., TSUKADA, M. \& SCHROTT, A., 2013 - Silver nanofilm sensors for assessing daguerreotype housing materials in an Oddy test setup. E-Preservation Science, 10: 71-76.

LECHTMAN, H. N. \& MACFARLANE, A. W., 2005 - La metalurgia del bronce en los Andes Sur Centrales: Tiwanaku y San Pedro de Atacama. Estudios Atacameños, 30: 7-27.

MIGNONE, P., 2015 - Illas y Allicac: La capacocha del Llullaillaco y los mecanismos de ascenso social de los "Inkas de privilegio". Boletín del Museo Chileno de Arte Precolombino, 20 (2): 69-87.

OBERHAUSER, F., FHURMANN, P. \& GAILLARD, M., 1957-1959 - Estudio metalúrgico. Boletín del Museo Nacional de Historia Natural, tomo 27 (1):78-81. Número temático titulado «La momia del Cerro El Plomo» (G. Mostny, ed.).

PETERSEN G., G., 1970 - Minería y metalurgia en el Antiguo Perú, 140 pp.; Lima: Arqueológicas, Museo Nacional de Antropología y Arqueología, vol. 12.

PHIPPS, E., 2015 - Inka textile traditions and their Colonial counterparts. In: The Inka Empire: A multidisciplinary approach (I. Shimada, ed.): 197- 236; Austin: University of Texas Press.

ROVIRA LLORÉNS, S. \& GÓMEZ RAMOS, P., 1995 - Los objetos de metal de la colección Juan Larrea: un estudio arqueometalúrgico. Anales del Museo de América, 3: 21-33. 
ROWE, J. H., 1996 - Inca. In: Andean art at Dumbarton Oaks, (E. Boon, ed.): 301-319; Washington D.C.: Dumbarton Oaks Research Library and Collection, vol. 1.

TISSOT, I., MONTEIRO, O. C., BARREIROS, M. A., CORREGIDOR, V., CORREIA, J. \& GUERRA, M. F., 2016 - Corrosion of silver alloys in sulphide environments: a multianalytical approach for surface characterization. Royal Society of Chemistry Advances, 57 (6): 51856-51863.

TROALEN, L., TATE, J. \& GUERRA, M. F. 2014 - Goldwork in Ancient Egypt. Workshop practices at Qurneh in the 2nd Intermediate Period. Journal of Archaeological Sciences, 50: 219-226.

VETTER, L., 2007 - La evolución del Tupu en forma y manufactura desde los Incas hasta el siglo XIX. In: Metalurgia en la América antigua. Teoría, arqueología y tecnología de los metales prehispánicos (R. Lleras Pérez, ed.): 101- 128; Bogotá: IFEA, Banco de la República, Fundación de Investigaciones Arqueológicas Nacionales.

VETTER, L. 2016 - Plateros y saberes Andinos: El arte orfebre en los siglos XVI-XVII, 326 pp.; Cuzco: Centro de estudios Bartolomé de las Casas, Academia Nacional de Ciencias/ ANC.

WATKINSON, D., 2010 - Preservation of metallic cultural heritage. In: Shreir's corrosion (T. J. A. Richardson, ed.): 3307-3340; Amsterdam: Elsevier Ltd., $4^{\text {th }}$ edition, vol. 4.

ZORI, C. M. \& TROPPER, P., 2010 - Late pre-Hispanic and early Colonial silver production in the Quebrada de Tarapacá, Northern Chile. Boletín del Museo Chileno de Arte Precolombino, 15: 65-87. 